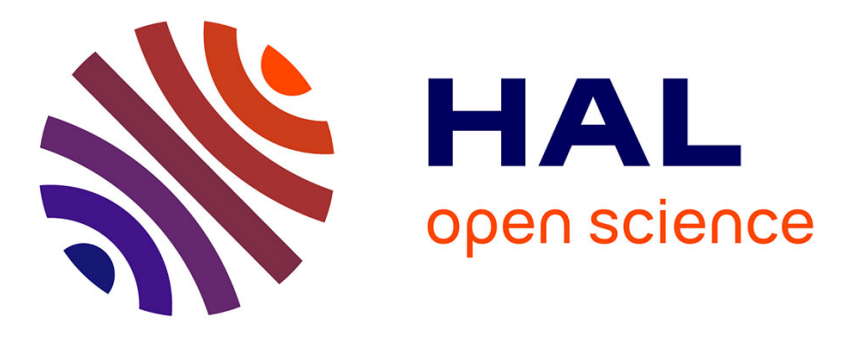

\title{
Robust Adhesive Precision Bonding in Automated Assembly Cells
}

\author{
Tobias Müller, Sebastian Haag, Thomas Bastuck, Thomas Gisler, Hansruedi \\ Moser, Petteri Uusimaa, Christoph Axt, Christian Brecher
}

\section{To cite this version:}

Tobias Müller, Sebastian Haag, Thomas Bastuck, Thomas Gisler, Hansruedi Moser, et al.. Robust Adhesive Precision Bonding in Automated Assembly Cells. 7th International Precision Assembly Seminar (IPAS), Feb 2014, Chamonix, France. pp.1-7, 10.1007/978-3-662-45586-9_1 . hal-01260713

\section{HAL Id: hal-01260713 https://hal.inria.fr/hal-01260713}

Submitted on 22 Jan 2016

HAL is a multi-disciplinary open access archive for the deposit and dissemination of scientific research documents, whether they are published or not. The documents may come from teaching and research institutions in France or abroad, or from public or private research centers.
L'archive ouverte pluridisciplinaire HAL, est destinée au dépôt et à la diffusion de documents scientifiques de niveau recherche, publiés ou non, émanant des établissements d'enseignement et de recherche français ou étrangers, des laboratoires publics ou privés. 


\title{
Title
}

Robust Adhesive Precision Bonding in Automated Assembly Cells

\section{Suggested topic area}

Precision joining operations

\section{Authors}

Tobias Müller ${ }^{1 *}$, Sebastian Haag ${ }^{1}$, Thomas Bastuck ${ }^{1}$, Thomas Gisler ${ }^{2}$, Hansruedi Moser ${ }^{2}$, Petteri Uusimaa $^{3}$, Christoph $\mathrm{Axt}^{4}$, Christian Brecher ${ }^{1}$

\author{
Affiliation \\ ${ }^{1}$ Fraunhofer Institute for Production Technology IPT, Steinbachstr. 17, 52074 Aachen, Germany \\ ${ }^{2}$ FISBA OPTIK AG, Rorschacher Str. 268, 9016 St. Gallen, Switzerland \\ ${ }^{3}$ Modulight Inc., Hermaniaku 22, 33720 Tampere, Finland \\ ${ }^{4}$ Rohwedder Micro Assembly GmbH, Opelstr. 1, 68789 St. Leon-Rot, Germany
}

\section{Contact details of corresponding author}

Tobias Müller

Fraunhofer Institute for Production Technology IPT

Steinbachstr. 17, 52074 Aachen, Germany

tobias.mueller@ipt.fraunhofer.de

Tel: +49-241-8904-493

Fax: +49-241-8904-6-493

\begin{abstract}
The assembly of optical components goes along with highest requirements regarding assembly precision. Laser products have become an integral part of many industrial, medical, and consumer applications and their relevance will increase significantly in the years to come. Still economic challenges remain. Assembly costs are driven by the demanding requirements regarding alignment and adhesive bonding. Especially in precision bonding are the interdependencies between alignment and bonding. Multiple components need to be aligned within smallest spatial and angular tolerances in submicron order of magnitude. A major challenge in adhesive bonding is the fact that the bonding process is irreversible. Accordingly, the first bonding attempt needs to be successful. Today's UVcuring adhesives inherit shrinkage effects during curing which are crucial for the submicron tolerances of e.g. FACs or beam combiners based on wavelength multiplexing what makes the bonding of these components very delicate assembly tasks. However, the shrinkage of UV-curing adhesives is not only varying between different loads due to fluctuations in raw materials, it is also changing along the storage period. An answer to this specific challenge can be the characterization of the adhesive on a daily basis. The characterization before application of the adhesive is necessary for precision optics assembly in order to reach highest output yields, minimal tolerances and ideal beamshaping results. The work presented in this paper aims for a significantly reduced impact of shrinkage effects during curing of highly durable UV-curing epoxy adhesives resulting in increased precision. Key approach is the highly precise volumetric dispensing of the adhesive as well as the characterization of the shrinkage level. These two key factors allow most reproducible adhesive bonding in automated assembly cells. These proceedings are essential for standardized automated assembly solutions which will prospectively play a major role in laser technology.
\end{abstract}




\section{Extended Abstract}

The assembly of optical components goes along with the highest requirements regarding assembly precision in many cases. For instance laser products have become an integral part of many industrial, medical, and consumer applications and their relevance will increase significantly in the years to come. Still, economic challenges of laser related products prevent the breakthrough in many applications. Assembly costs are driven mainly by the demanding requirements regarding alignment and adhesive bonding in all spatial dimensions. Extensive research efforts are being made in matters of novel bonding methods, whilst the challenges of the state-of the art UV-curing adhesive bonding still provide opportunities for improvement as identified in several recent research projects and contributions ([1],[2],[3],[4],[5],[6],[7]).

The major and most appreciated advantages of UV-curing adhesive bonding are its low temperature, the automation friendliness, and the reasonable costs for automation solutions. Yet, especially challenging in precision assembly are the interdependencies between alignment and adhesive bonding. Shrinkage effects during curing of the adhesives are crucial for the submicron tolerances of for instance fast-axis collimators (FACs). Also beam combiners based on wavelength multiplexing are a very delicate assembly task. Multiple components sharing interdependencies need to be aligned within smallest tolerances in submicron order of magnitude. For such assembly tasks the major challenge in adhesive bonding at highest precision level is the fact, that the bonding process is irreversible. Accordingly, the first bonding attempt needs to be successful as especially for automated solutions dissolving the link between two components is not possible. The impact of the shrinkage effects can be tackled both by a suitable design of the bonding area and a positioning offset of the optic for compensation purposes. Yet, compensating shrinkage effects is difficult, as the shrinkage of UVcuring adhesives is not necessarily constant between two different loads due to fluctuations in raw materials and varies over the storage period even under ideal circumstances an up-to-date characterization of the adhesive is necessary for automation in optics assembly to reach highest output yields, minimal tolerances and ideal beam-shaping results. Accordingly, today the operator needs to adjust the compensation offset data on a daily basis during the first assembly processes of the production series as practice shows. Chances of creating sub-standard goods are high for these first systems, which is problematic for high-value precision optics.

The work presented in this paper aims for a significantly reduced impact of shrinkage effects of UV curing adhesives and a resulting increase in precision in automated assembly cells. Key approach is the highly precise volumetric dispensing of the adhesive as well as an up-to-date characterization of the shrinkage level. These two key factors allow reproducible adhesive precision bonding in automated assembly cell.

First step in the approach for increased precision in adhesive bonding is to model the bonding system and identify and separate the origins for the misalignment effects in order to be able to compensate the shrinkage effects more efficiently. The model of the bonding area that was elaborated presumes that the misalignment is mainly caused by two effects: A spatial and an angular offset. Figure 1 shows the model of the bonding area.

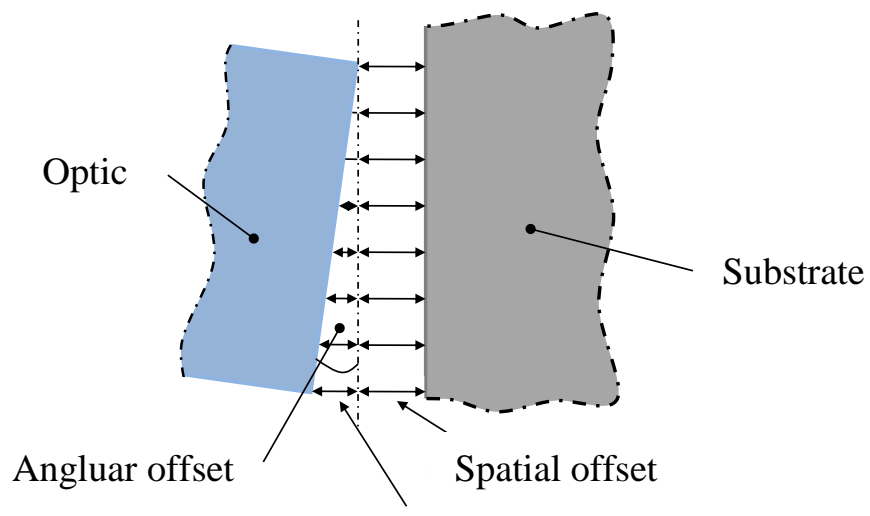

Total offset

Figure 1: Model for compensation of shrinkage effects 
Spatially, the bonding area consists of an offset part which is causing a linear shrinkage in normal direction with reference to the substrate surface. This shrinkage effect is less crucial, as kinematically it leads to small movements which can be compensated linear. The bigger impact on the bonding area has the second wedge-shaped part as it leads to angular misalignments causing an angular $(\beta)$ and lateral $(\Delta x)$ displacement of the component due to the kinematic shown in Figure 2.

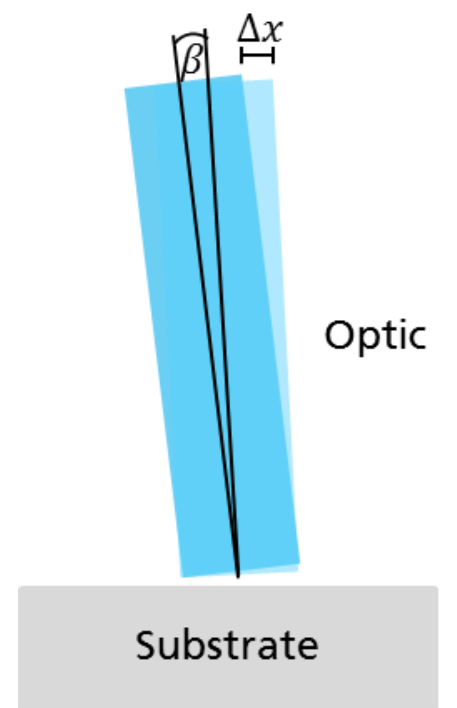

\section{Figure 2: Kinematics for misalignment caused by wedge-shaped bonding area}

For optical components these angular (tip-tilt) misalignments are in many cases the most sensitive degrees of freedom. Whilst interferometrical measurements allow a reliable and precise measurement of the spatial misalignments, for the angular misalignments these measurements are more complex and demanding.

In order to determine the volumetric shrinkage during curing of the adhesive a measurement setup was built. The measurement principle is depicted in Figure 3. In order to quantify shrinkage effects a defined angle between the two bonding partners was set up. This predefined misalignment causes a wedge shaped gluing area. As the glue is cured, it shrinks and causes a misalignment of the optical component. The motion of the glass cube is monitored and measured by a PSD. Essential in order to create reliable and reproducible measurement results is the precise verification of the angle $\beta$.

Therefore telecentric camera was setup and carefully aligned to the testing setup in order to measure the edges of the two components. Using an image processing routine the angle could be precisely set utilizing a micromanipulator holding the glasscube and manipulating it in the necessary degrees of freedom. The Micromanipulator used was the flexure based Commander 6 which has been developed at Fraunhofer IPT.

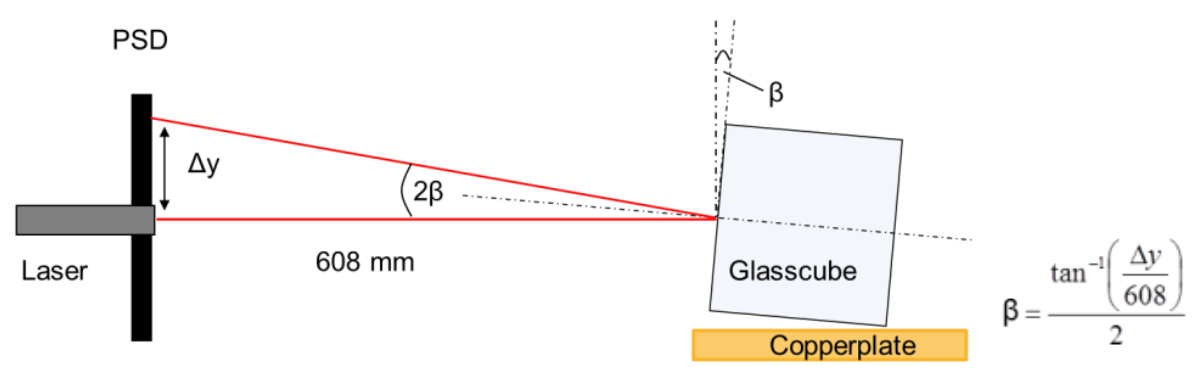

Figure 3: Principle measurement setup for shrinkage quantification

With this measurement setup it was possible to determine angular movements with a precision of less than $10 \mu \mathrm{rad}$ covering a measurement range of up to $10^{\circ}$. Figure 4 shows an exemplary measurement of the shrinkage effects. The wedge angle in this measurement was $4.36^{\circ}$ the spatial offset of $100 \mu \mathrm{m}$. 


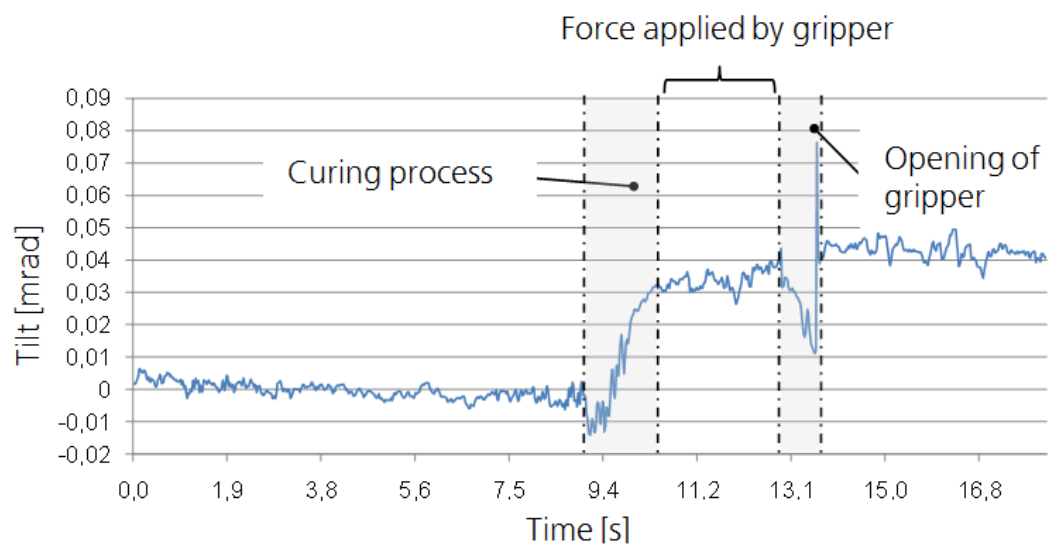

Figure 4: Tilt measurement sample of curing process

By using a highspeed-PSD device it was possible to acquire the motion data in realtime which provides information about the temporal shrinkage behaviour during the curing process. Remarkable is a minor expansion at the beginning of the curing process followed by a fast expansion until the final curing result is reached. Opening the mechanical gripper leads to force amplitudes in both directions and a final relaxation leading to an increase of the tilt by about $10 \mu \mathrm{rad}$. The quantification and variation of the gripper's closing force force allowed an approximation of the forces induced by the adhesive during curing process. This knowledge is essential for bonding process development when the components shall be kept in place by the application of a retaining force. Therefore the gripper was actuated pneumatically allowing a precise an easy variation of the clamping force.

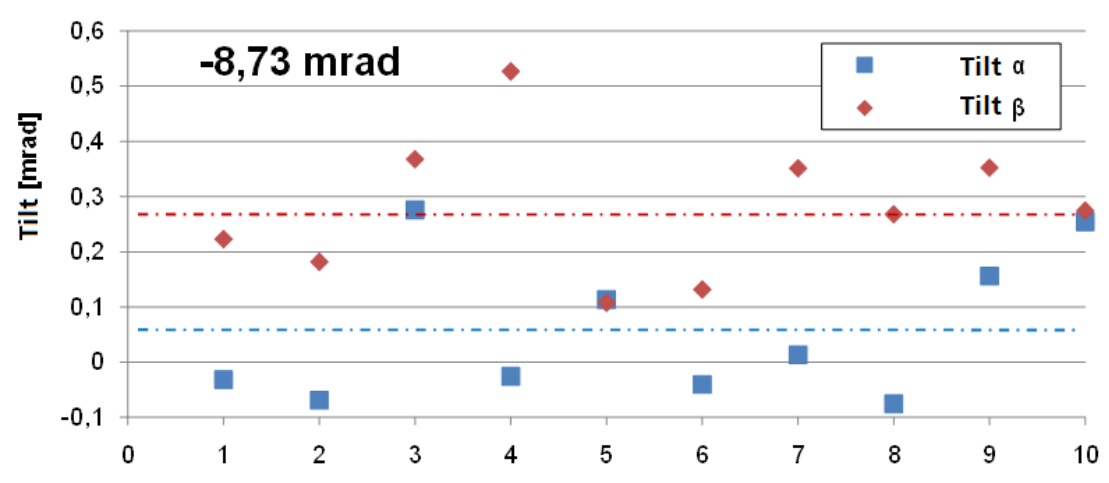

Figure 5: Tilt measurement sample of curing process

Representative results for a measurement of one sample adhesive are shown in Figure 5. The initial wedge angle is this specific case was $8,73 \mathrm{mrad}\left(-0,5^{\circ}\right)$. The mean tilt resulting from curing in the designated shrink direction (Tilt $\beta$ ) was at $0,287 \mathrm{mrad}$. The undesired parasitic tilt movement (Tilt $\alpha$ ) was at $0,0596 \mathrm{mrad}$ which represents the measuring uncertainty. Formula (1) is describing the linear shrinkage neglecting the linear shrinkage resulting from parallel movements of the components.

$$
\begin{aligned}
& \Delta \alpha=-\alpha \cdot l S \\
& \Delta \alpha=\text { Tilt induced by shrinkage } \\
& \alpha=\text { Adjusted wedge-angle } \\
& l S=\text { linear shrinkage }
\end{aligned}
$$

The corresponding shrinkage in this experiment was $2,31 \%$ which is roughly double the value given in the technical data sheet. This deviation is caused by ageing effects of the adhesives during their shelf 
life. Over the time the UV-curable adhesives partially react even without any exposure to UV-light. Accordingly, the magnitude of shrinkage drops with the age of the adhesive.

However, the severity of shrinkage is not the only factor influencing the achievable bonding results. Also highly precise volumetric dosing and minimal adhesive volumes minimize the shrinkage effects. Therefore a qualification methodology for dosing systems has been elaborated allowing an efficient selection of the best suitable dosing system. Evaluation criteria are minimum dispensing volume, reproducibility, handling and costs. Jet dosing systems prove to be most reliable regarding dispensing volume reproducibility. A further increase in precision of volumetric dispensing could be achieved by creating minimal dropsizes and applying several drops to generate one drop of the desired volume. Once again, a telecentric camera and an image processing routine were used to characterize the reproducibility of the drop diameter. Based on these results a number of suitable adhesives for bonding optics has been validated and characterized regarding shrinkage developments over their lifetime.

Result of the work presented in the paper is the precise predictive compensation of shrinkage effects of UV-curing adhesives during the curing process. Firstly, the importance of precise volumetric dosing was elaborated. Furthermore the impact of shrinkage effects has been modelled, described and quantified. The determination of the volumetric shrinkage magnitude is possible with the measurement setup described. The shrinkage could be determined as precisely a $0,1 \%$. During the experimental phase shifts in shrinkage of the same adhesive of more than 5\% due to ageing effects were observed. For precise and efficient compensation of the shrinkage effects a measurement of the shrinkage magnitude instantly before the glue application appears to be unavoidable. The determination of the shrinkage value is even more important in automated assembly solutions, as the offset values can thus be calculated dynamically.

Looking ahead automation will play a growingly important role in laser optics assembly. For robust automation solutions in optics assembly producing best results the detailed knowledge of the adhesives behaviour as well as a sophisticated compensation of the shrinkage is obligatory. Accordingly, the proceedings presented in this paper are essential for standardized automated assembly solutions. 


\section{References}

[1] E.Beckert; T.Burkhardt; R.Eberhardt; A.Tünnermann (2008):

Solder Bumping - a flexible Joining Approach for the Precision Assembly of optoelectronical Systems. Micro-Assembly Technologies and Applications. Ifip Tc5 Wg5.5 Fourth International Precision Assembly Seminar (Ipas'2008) Chamonix, France, February 10-13, 2008. Proceedings: Springer, pp.139-148

[2] J. Miesner, A. Timmermann, J. Meinschien, B. Neumann, S. Wright, T. Tekin, H. Schröder, T. Westphalen, F. Frischkorn: Automated Assembly of Fast Axis Collimation (FAC) Lenses for Diode Laser Bar Modules Photonics West 2009, San Jose, 26. January 2009, PW09L-LA1077198-15 Wash: Proc. SPIE 7198, 71980G (2009); doi:10.1117/12.809190

[3] Brecher, C.; Pyschny, N.; Haag, S.; Guerrero Lule, V. (2012): Automated alignment of optical components for high-power diode lasers. In: Mark S. Zediker (Hg.): High-power diode laser technology and applications X. 22-24 January 2012, San Francisco, California, United States. Bellingham, Wash: SPIE (Proceedings of SPIE, v. 8241), S. 82410D-82410D-11.

[4] Brecher, C.; Pyschny, N.; Haag, S.; Mueller, T. (2013): Automated assembly of VECSEL components. In: Jennifer E. Hastie (Hg.): Vertical external cavity surface emitting lasers (VECSELs) III. 3-5 February 2013, San Francisco, California, United States (Proceedings of SPIE, volume 8606), S. 86060I-86060I-14.

[5] Garlich, Torsten; Guerrero, Vicente; Hoppen, Martin; Müller, Tobias; Pont, Pere; Pyschny, Nicolas et al. (2012): SCALAB. Scalable Automation for Emerging Lab Production. Final report of the MNT-ERA.net research project. 1. ed. Aachen: Apprimus-Verl.

[6] Miesner, Jörn; Timmermann, Andre; Meinschien, Jens; Neumann, Bernhard; Wright, Steve; Tekin, Tolga et al. (2009). In: Mark S. Zediker (Hg.): High-power diode laser technology and applications VII. 26-27 January 2009, San Jose, California, United States. Bellingham, Wash: SPIE (v. 7198), S. 71980G-71980G-11.

[7] Pierer, J.; Lützelschwab, M.; Grossmann, S.; Spinola Durante, G.; Bosshard, Ch.; Valk, B. et al. (2011). In: Mark S. Zediker (Hg.): High-power diode laser technology and applications IX. 2325 January 2011, San Francisco, California, United States. Bellingham, Wash: SPIE (v. 7918), S. 79180I-79180I-8. 